\title{
Lowered intraocular pressure in a glaucoma patient after intravitreal injection of ocriplasmin
}

This article was published in the following Dove Press journal:

Clinical Ophthalmology

23 October 2015

Number of times this article has been viewed

\author{
Michael McClintock' \\ Mathew W MacCumber ${ }^{1,2}$ \\ 'Department of Ophthalmology, Rush \\ University Medical Center, ${ }^{2}$ lllinois \\ Retina Associates, S.C., Chicago, \\ IL, USA
}

\begin{abstract}
We report the case of a glaucoma patient who received a single intravitreal injection of $125 \mu \mathrm{g}$ ocriplasmin for vitreomacular traction in the right eye. The patient had bilateral advanced glaucoma and had previously undergone an implantation of an Ahmed glaucoma valve in the right eye and trabeculectomy in both eyes. The patient was using three topical ophthalmic intraocular pressure (IOP)-lowering medications on the day of injection. Baseline uncorrected Snellen visual acuity was 20/80-1 and IOP was $19 \mathrm{mmHg}$. Resolution of vitreomacular traction was achieved 1 week after injection. IOP was transiently decreased, reaching a maximum reduction of $12 \mathrm{mmHg}$ below baseline at 1 month after injection, when serous choroidal effusion was also present. IOP returned to baseline levels and choroidal effusion resolved at 2 months after injection of IOP-lowering medication. Vitrectomy with epiretinal membrane and internal limiting membrane peeling, endolaser photocoagulation, and fluid-gas exchange were performed in the right eye $\sim 3.5$ months after injection to treat persistent epiretinal membrane, and presumed tractional retinal detachment. Final visual acuity was $20 / 50+$ and IOP was $18 \mathrm{mmHg}$ at 16 weeks after surgery. To our knowledge, this is the first report of IOP reduction and serous choroidal effusion after ocriplasmin injection.
\end{abstract}

Keywords: ocriplasmin, intraocular pressure, vitrectomy, choroidal effusion

\section{Introduction}

Vitreomacular traction (VMT) is a sight-threatening condition that can lead to reduced visual acuity (VA) and metamorphopsia. ${ }^{1,2}$ Intraocular pressure (IOP) deviations are not known to be associated with VMT. Current VMT management strategies include observation, vitrectomy, and pharmacologic vitreolysis with ocriplasmin (recombinant truncated human plasmin). ${ }^{1,3}$ The safety and efficacy of a single intravitreal injection of $125 \mu \mathrm{g}$ ocriplasmin for the resolution of symptomatic vitreomacular adhesion (including VMT) has been demonstrated. ${ }^{1,2}$ A significantly greater proportion of patients receiving ocriplasmin versus (vs) vehicle achieved resolution of vitreomacular adhesion $(26.5 \%$ vs $10.1 \%, P<0.001){ }^{2}$ No substantially reduced IOP was reported, and the proportions of patients with increased IOP were similar between groups. ${ }^{2}$ We report the case of a glaucoma patient with VMT who experienced transiently lowered IOP and choroidal effusion after ocriplasmin injection.

\section{Case description}

Approval was received from the patient and Illinois Retina Associates to access the patient's medical records. Rush University Medical Center reviewed and considered the study Institutional Review Board exempt. Dr McClintock presented this study at Rush University Medical Center.

A 77-year-old Caucasian male with bilateral advanced glaucoma previously underwent cataract extraction with posterior chamber intraocular lens implantation in both 
eyes. An neodymium-doped yttrium aluminum garnet capsulotomy was subsequently performed in the right eye, and an Ahmed glaucoma valve was implanted to manage high IOP (average $28 \mathrm{mmHg}$ ). The patient had been initially referred to us at this time for VMT with concomitant epiretinal membrane (ERM) in the right eye. With uncorrected VA 20/40 in the right eye, the patient opted for observation. Initially there was a reduction in IOP, but by 4 weeks after glaucoma surgery the IOP returned to preoperative levels. During the observation period, a bleb needling over the plate was attempted, without significant success. A month following, both eyes underwent trabeculectomy with mitomycin C. After the trabeculectomy, the IOP ranged from 18 to $22 \mathrm{mmHg}$ while on three topical glaucoma (IOP-lowering) medications: dorzolamide, brimonidine, and bimatoprost. Progressive VA decline in the right eye attributable to worsening VMT prompted the patient to request ocriplasmin.

On the day of injection, VA was 20/80-1 in the right eye (Figure 1A) and 20/20-1 in the left eye. IOP was 19 $\mathrm{mmHg}$ in the right eye and $10 \mathrm{mmHg}$ in the left eye. A preinjection exam showed a deep anterior chamber in the right eye, a superotemporal tube without visible obstruction, patent peripheral iridotomy superonasally, and posterior chamber intraocular lens with open posterior capsule. The posterior segment had trace vitreous hemorrhage inferiorly (1 week before injection); no retinal breaks were visible before or after the injection with careful scleral depression. The patient experienced photopsias in the right eye post injection.

At 1 week post injection, VMT was resolved and VA was 20/70-1 (Figure 1B). IOP decreased $8 \mathrm{mmHg}$ to a final reading of $11 \mathrm{mmHg}$. At 1 month post injection, flashing lights were reported in the temporal periphery. VA was 20/70-2 and IOP decreased to $7 \mathrm{mmHg}$ (Figure 1C). All glaucoma medications were discontinued in the right eye, and atropine and prednisolone were initiated. The anterior chamber was stable and deep, with Seidel-negative bleb. There was no obvious change in the trabeculectomy bleb morphology after the injection. There did appear to be elevation of the bleb over the plate following the injection; however, the conjunctiva appeared thickened. There was serous choroidal effusion and inferior retinal detachment (Figure 1D), with no visible retinal break.

At 2 months post injection, VA was 20/60-1 and IOP increased to $17 \mathrm{mmHg}$ (Figure 2A). Choroidal effusion resolved. Retinal detachment persisted inferiorly (Figure 2B),
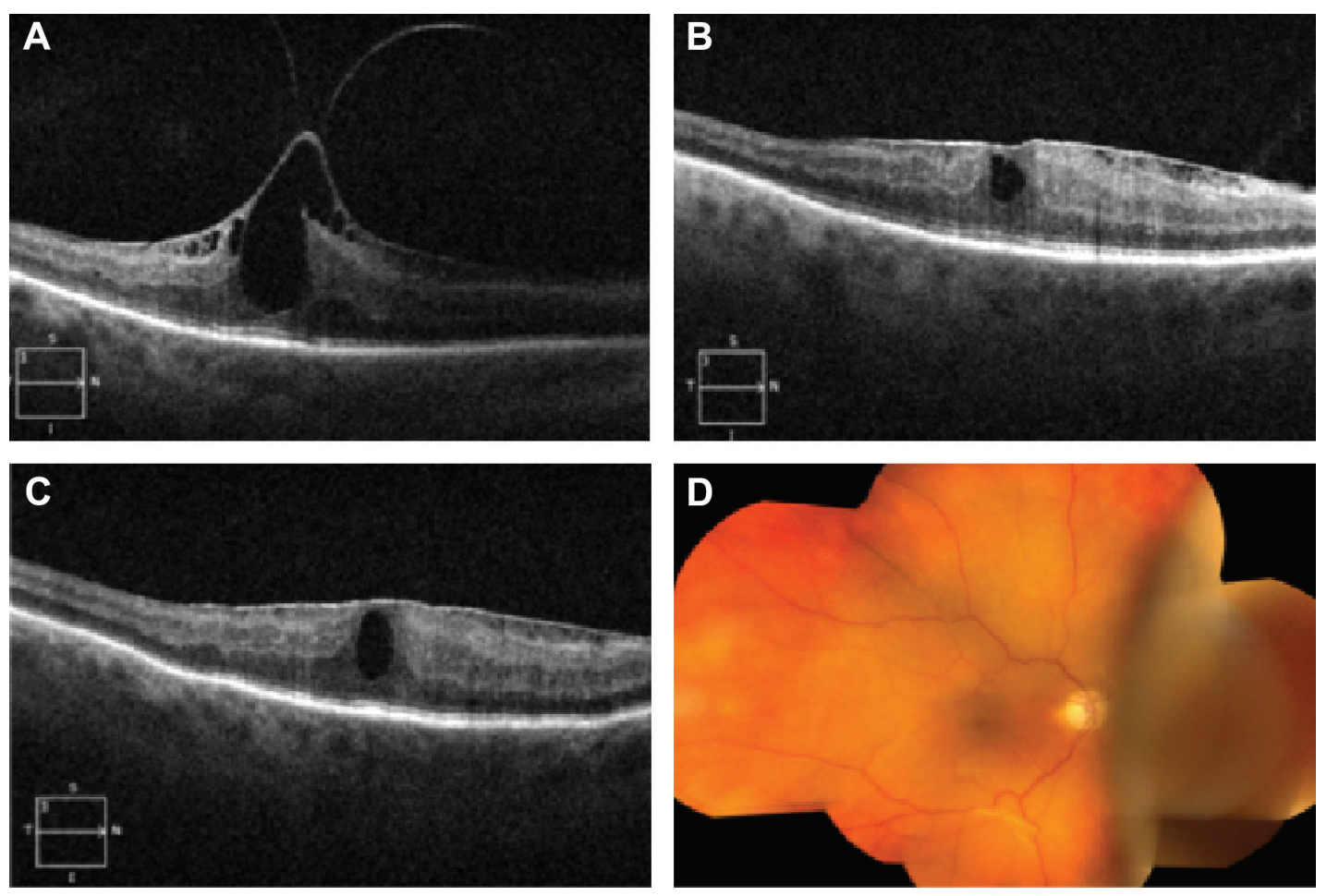

Figure I Vitreomacular traction (VMT) resolution, lowered intraocular pressure (IOP), and choroidal effusion after ocriplasmin injection.

Notes: (A) Optical coherence tomography (OCT) scan through the fovea from before injection shows VMT. Visual acuity was 20/80-I, IOP was I9 mmHg. (B) OCT scan I week after injection shows clear epiretinal membrane (ERM). Visual acuity was 20/70-I, IOP was II mmHg. (C) One month after injection, OCT scan shows clear resolution of VMT but remaining ERM. Visual acuity was 20/70-2, IOP decreased to $7 \mathrm{mmHg}$. (D) Fundus photograph from 1 month after injection shows nasal choroidal effusion and inferior retinal detachment. 

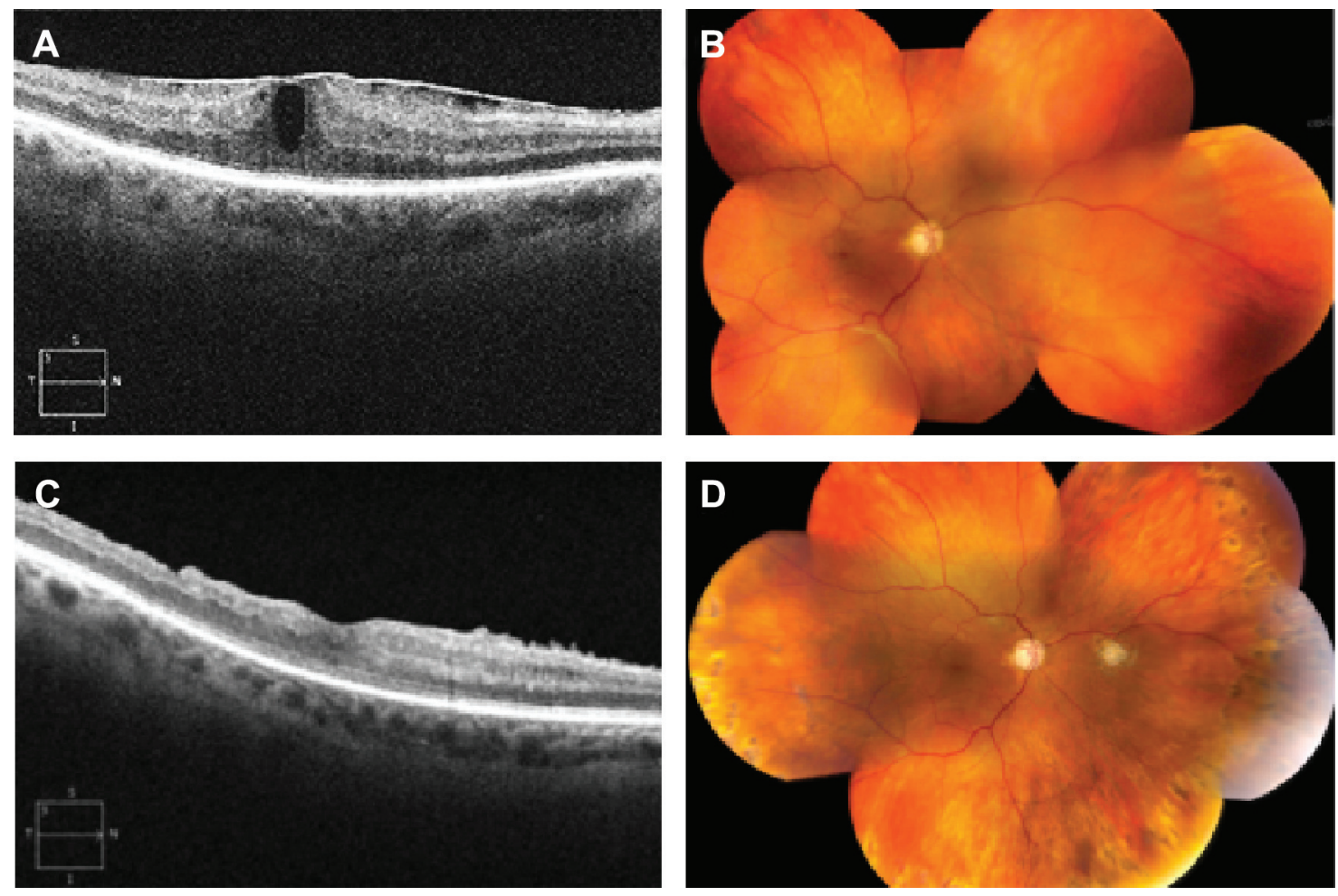

Figure 2 Choroidal effusion resolved and retinal detachment persisted at 2 months following ocriplasmin injection.

Notes: (A) Two months after injection, optical coherence tomography (OCT) scan still shows remaining epiretinal membrane (ERM). Visual acuity was 20/60-I, intraocular pressure (IOP) increased to $17 \mathrm{mmHg}$. (B) Fundus photograph from 2 months after injection shows choroidal effusion resolved and persistent inferior retinal detachment. (C) OCT scan from 16 weeks after vitrectomy shows absent ERM. Visual acuity was 20/50+, and IOP was 18 mmHg. (D) Fundus photograph from I6 weeks after vitrectomy shows retinal reattachment.

with peripheral vitreous attachment noted outside of the inferotemporal arcade. The patient requested vitrectomy in the right eye to treat persistent ERM and retinal detachment, presumed tractional (ie, significant vitreous traction was seen both preoperatively and at time of vitrectomy, in addition to no visible retinal breaks).

A vitrectomy was performed approximately 3.5 months post injection. During surgery, vitreoretinal adhesion was noted for 360 degrees in the near periphery. The retinal detachment extended only to the equator and no retinal break was found in the detached retina. A small retinal hole was identified along the superotemporal arcade in attached retina. The ERM and internal limiting membrane were peeled, and retina reattached with 360-degree endolaser photocoagulation and fluid-gas exchange. At 16 weeks post surgery (Figure 2C, D), VA was 20/50+, and IOP was $18 \mathrm{mmHg}$ with the patient on no glaucoma medications.

\section{Discussion}

To our knowledge, this is the first case report of IOP decrease and choroidal effusion after ocriplasmin injection. IOP was higher in the right eye vs the left eye before injection, despite treatment with IOP-lowering medications and a glaucoma drainage device in the right eye. Occluded devices can lead to IOP increases. Ocriplasmin is a recombinant truncated form of the human enzyme plasmin that has proteolytic effects on fibrin, fibrinogen, fibronectin, collagen, and laminin, ${ }^{2,3}$ and may have lowered IOP by degrading vitreous or connective tissue, in a hypothetically occluded device or the surrounding fibrous capsular bleb, resulting in serous choroidal effusion and retinal detachment. For instance, there may have been lysis of a small non-visible fibrin clot from hemorrhage, or vitreous or other proteinaceous material occluding the glaucoma drainage tube. There is also a possibility that the enzymatic activity may have increased the permeability of the pseudocapsule around the drainage implant, leading to decreased IOP. Previous reports support a role for plasminmediated proteolysis in clearing occluded glaucoma drainage devices. ${ }^{4,5}$ In addition, persistent peripheral vitreoretinal traction likely contributed to the persistent retinal detachment. It is unlikely that the tractional retinal detachment caused the low IOP, which is usually caused by rhegmatogenous retinal detachments. Given that there were no identifiable retinal breaks in the detached retina, aqueous humor would likely not have had access to the subretinal space. If the decrease in IOP was due to the detachment, one would expect the IOP to 
have increased following the repair of the detachment, which did not occur. In summary, advanced glaucoma patients with VMT should be carefully monitored for IOP changes after intravitreal injection of ocriplasmin.

\section{Acknowledgments}

Writing support provided by ThromboGenics and executed by Meridius Health Communications.

\section{Author contributions}

All authors contributed toward data analysis, drafting and revising the paper and agree to be accountable for all aspects of the work.

\section{Disclosure}

Dr MacCumber reported receiving consultant fees from Allergan, Genentech, Optos, Regeneron, ThromboGenics and receiving grant support from Allergan, ArcticDx, Regeneron, Sequenom, and ThromboGenics. Dr McClintock reported no conflicts of interest.

\section{References}

1. Jackson TL, Nicod E, Simpson A, Angelis A, Grimaccia F, Kanavos P. Symptomatic vitreomacular adhesion. Retina. 2013;33(8):1503-1511.

2. Stalmans P, Benz MS, Gandorfer A, et al. Enzymatic vitreolysis with ocriplasmin for vitreomacular traction and macular holes. N Engl J Med. 2012;367(7):606-615.

3. JETREA [package insert]. Iselin, NJ: ThromboGenics, Inc.; 2014.

4. Tsui I, Airiani S, Wen A, El-Sawy T, Fine HF, Maris PJ Jr. Intravitreal injection of tissue plasminogen activator as treatment for an occluded pars plana glaucoma tube. Clin Ophthalmol. 2009;3:91-93.

5. Zalta AH, Sweeney CP, Zalta AK, Kaufman AH. Intracameral tissue plasminogen activator use in a large series of eyes with valved glaucoma drainage implants. Arch Ophthalmol. 2002;120(11):1487-1493.
Clinical Ophthalmology

\section{Publish your work in this journal}

Clinical Ophthalmology is an international, peer-reviewed journal covering all subspecialties within ophthalmology. Key topics include: Optometry; Visual science; Pharmacology and drug therapy in eye diseases; Basic Sciences; Primary and Secondary eye care; Patient Safety and Quality of Care Improvements. This journal is indexed on

Submit your manuscript here: http://www.dovepress.com/clinical-ophthalmology-journal

\section{Dovepress}

PubMed Central and CAS, and is the official journal of The Society of Clinical Ophthalmology (SCO). The manuscript management system is completely online and includes a very quick and fair peer-review system, which is all easy to use. Visit http://www.dovepress.com/ testimonials.php to read real quotes from published authors. 